\title{
Relativistic Stars in Massive Gravity
}

\author{
Masashi Yamazaki ${ }^{\star a}$, Taishi Katsuragawa, ${ }^{a}$ Shin'ichi Nojiri, ${ }^{a b}$ and Sergei. D. \\ Odintsov, ${ }^{\text {cde }}$ \\ ${ }^{a}$ Department of Physics, Nagoya University \\ 464-8602, Nagoya, Japan \\ ${ }^{b}$ Kobayashi-Maskawa Insttute for the Origin of Particles and the Universe \\ 464-8602, Nagoya, Japan \\ ${ }^{c}$ Institut de Ciencies de lEspai (IEEC-CSIC), Campus UAB \\ Carrer de Can Magrans, s/n, 08193 Cerdanyola del Valles, Barcelona, Spain \\ ${ }^{d}$ ICREA, Passeig LluAs Companys \\ 23, 08010 Barcelona, Spain \\ ${ }^{e}$ Tomsk State Pedagogical University \\ 634061 Tomsk, Russia \\ E-mail: taishidth.phys.nagova-u.ac.jp, \\ nojiridgravity.phys.nagoya-u.ac.jp, dintsovdice.cat
}

\begin{abstract}
Maximal mass of relativistic star is one of the quantities which may distinguish modified gravity theories because it is strongly influenced by the short-range behavior of gravitation. We derive modified Tolman-Oppenheimer-Volkoff equations for the de Rham-Gabadadze-Tolley (dRGT) massive gravity with the flat reference metric. We find that new constraint equations appear and that the maximum masses in the minimal model of dRGT massive gravity become smaller than those in the general relativity.
\end{abstract}

The 3rd International Symposium on "Quest for the Origin of Particles and the Universe" 5-7 January 2017

Nagoya University, Japan

\footnotetext{
* Speaker.
} 


\section{Introduction}

Modified gravity, which is one of alternative theories of general relativity, has been considered for explaining the late-time accelerated expansion of the Universe without cosmological constant. In addition to such long-range physics, the short range properties should be also considered. The maximal mass of relativistic stars is one of quantities which is strongly influenced by the short range properties because it is determined by gravity and pressure from the matters inside the stars.

Recently, massive neutron stars have been discovered. A previous research shows that some models of modified gravity theories could explain such a massive neutron stars and predict heavier relativistic stars than those in the general relativity [四]. Therefore, the differences between modified gravity theories and the general relativity can be found significantly in large curvature or strong gravitational field phenomena. In this work, we consider the de Rham-Gabadadze-Tolley (dRGT) massive gravity that describes a ghost-free massive spin-2 particle, and investigate the effects of finite graviton mass to massive relativistic stars.

\section{Modified TOV equations}

The action of dRGT massive gravity [] $]$ is given by

$$
S_{\mathrm{dRGT}}=\frac{1}{2 \kappa^{2}} \int \mathrm{d}^{4} x \sqrt{-\operatorname{det}(g)}\left[R(g)-2 m_{0}^{2} \sum_{n=0}^{4} \beta_{n} e_{n}\left(\sqrt{g^{-1} f}\right)\right]+S_{\mathrm{mat}} .
$$

Here $\kappa^{2}=8 \pi G$ is gravitational constant and $m_{0}$ and $\beta_{n}$ are free parameters in this theory. The symmetric tensor $f_{\mu \nu}$ is called as reference metric which is non-dynamical. The square root is defined by

$$
\left(\sqrt{g^{-1} f}\right)^{\mu} \rho\left(\sqrt{g^{-1} f}\right)^{\rho}=g^{\mu \rho} f_{\rho v}
$$

and the polynomials are given as follows,

$$
\begin{aligned}
& {[\mathbf{X}]=X_{\mu}^{\mu}, \quad e_{0}(\mathbf{X})=1, \quad e_{1}(\mathbf{X})=[\mathbf{X}], \quad e_{2}(\mathbf{X})=\frac{1}{2}\left([\mathbf{X}]^{2}-\left[\mathbf{X}^{2}\right]\right),} \\
& e_{3}(\mathbf{X})=\frac{1}{6}\left([\mathbf{X}]^{3}-3[\mathbf{X}]\left[\mathbf{X}^{2}\right]+2\left[\mathbf{X}^{3}\right]\right), \\
& e_{4}(\mathbf{X})=\frac{1}{24}\left([\mathbf{X}]^{4}-6[\mathbf{X}]^{2}\left[\mathbf{X}^{2}\right]+3\left[\mathbf{X}^{2}\right]^{2}+8[\mathbf{X}]\left[\mathbf{X}^{3}\right]-6\left[\mathbf{X}^{4}\right]\right)=\operatorname{det}(\mathbf{X}), \\
& e_{k}(\mathbf{X})=0 \quad \text { for } \quad k>4
\end{aligned}
$$

The equations of motion are

$$
\begin{aligned}
0= & R_{\mu v}(g)-\frac{1}{2} R(g) g_{\mu \nu} \\
& +\frac{1}{2} m_{0}^{2} \sum_{n=0}^{3}(-1)^{n} \beta_{n}\left[g_{\mu \lambda} Y_{(n) v}^{\lambda}\left(\sqrt{g^{-1} f}\right)+g_{v \lambda} Y_{(n) \mu}^{\lambda}\left(\sqrt{g^{-1} f}\right)\right]-\kappa^{2} T_{\mu \nu} .
\end{aligned}
$$


And the matrices $Y$ are defined by

$$
\begin{aligned}
& Y_{(n) v}^{\lambda}(\mathbf{X})=\sum_{r=0}^{n}(-1)^{r}\left(X^{n-r}\right)^{\lambda}{ }_{v} e_{r}(\mathbf{X}), \\
& Y_{0}(\mathbf{X})=\mathbf{1}, \quad Y_{1}(\mathbf{X})=\mathbf{X}-\mathbf{1}[\mathbf{X}], \quad Y_{2}(\mathbf{X})=\mathbf{X}^{2}-\mathbf{X}[\mathbf{X}]+\frac{1}{2} \mathbf{1}\left([\mathbf{X}]^{2}-\left[\mathbf{X}^{2}\right]\right), \\
& Y_{3}(\mathbf{X})=\mathbf{X}^{3}-\mathbf{X}^{2}[\mathbf{X}]+\frac{1}{2} \mathbf{X}\left([\mathbf{X}]^{2}-\left[\mathbf{X}^{2}\right]\right)-\frac{1}{6} \mathbf{1}\left([\mathbf{X}]^{3}-3[\mathbf{X}]\left[\mathbf{X}^{2}\right]+2\left[\mathbf{X}^{3}\right]\right), \\
& Y_{(n) v}^{\lambda}(\mathbf{X})=0, \quad n \geq 4 .
\end{aligned}
$$

If the $\sqrt{g^{-1} f}$ is symmetric, the equations of motion can be written in a similar way

$$
\begin{aligned}
& G_{\mu v}+m_{0}^{2} I_{\mu \nu}=\kappa^{2} T_{\mu v} \\
& I_{\mu \nu}=\sum_{n=0}^{3}(-1)^{n} \beta_{n} g_{\mu \lambda} Y_{(n) v}^{\lambda}\left(\sqrt{g^{-1} f}\right) .
\end{aligned}
$$

The divergence of $g_{\mu v}$ leads new constraints $\nabla_{\mu} I^{\mu v}=0$ because of the Bianchi identities and energy-momentum conservation.

To obtain spherical and static solutions in flat reference metric, we use following ansatz

$$
\begin{aligned}
& g_{\mu \nu} \mathrm{d} x^{\mu} \mathrm{d} x^{\nu}=-A(\chi) \mathrm{d} t^{2}+B(\chi) \mathrm{d} \chi^{2}+D(\chi)^{2}\left(\mathrm{~d} \theta^{2}+\sin ^{2} \theta \mathrm{d} \phi^{2}\right) \\
& f_{\mu \nu} \mathrm{d} x^{\mu} \mathrm{d} x^{\nu}=-\mathrm{d} t^{2}+\mathrm{d} \chi^{2}+\chi^{2}\left(\mathrm{~d} \theta^{2}+\sin ^{2} \theta \mathrm{d} \phi^{2}\right) .
\end{aligned}
$$

For convenience, we use redefined functions $A(\chi)=e^{2 v(r)}$ and $B(\chi) \chi^{\prime}(r)^{2}=e^{2 \lambda(r)}$. Moreover $\chi \equiv \chi(r)$ is used as the inverse function of $D(\chi) \equiv r^{2}$. Therefore the ansatz ([2]) can be rewritten as

$$
\begin{aligned}
& g_{\mu \nu} d x^{\mu} d x^{\nu}=-e^{2 v(r)} d t^{2}+e^{2 \lambda(r)} d r^{2}+r^{2}\left(d \theta^{2}+\sin ^{2} \theta d \phi^{2}\right), \\
& f_{\mu \nu} d x^{\mu} d x^{\nu}=-h(r) d t^{2}+h(r)^{-1}\left(\chi^{\prime}(r)\right)^{2} d r^{2}+\chi(r)^{2}\left(d \theta^{2}+\sin ^{2} \theta d \phi^{2}\right) .
\end{aligned}
$$

The function $\chi(r)$ is determined by the new constraints $\nabla_{\mu} I^{\mu v}=0$.

The modified TOV equations are given by

$$
\begin{aligned}
& G M^{\prime}=4 \pi G \rho r^{2}+\frac{m_{0}^{2}}{2} r^{2} I_{t}^{t}, \quad-\frac{p^{\prime}}{p+\rho}=v^{\prime}=\frac{4 \pi G p r^{3}+G M-\frac{m_{0}^{2}}{2} r^{3} I_{r}^{r}}{r(r-2 G M)}, \\
& \kappa^{2} p=\left(v^{\prime \prime}+v^{\prime 2}+\frac{v^{\prime}}{r}\right)\left(1-\frac{2 G M}{r}\right)+\frac{1}{2}\left(\frac{1}{r}+v^{\prime}\right)\left(1-\frac{2 G M}{r}\right)^{\prime}+m_{0}^{2} I_{\theta}^{\theta},
\end{aligned}
$$


and $I^{\mu}{ }_{v}$ are explicitly given by

$$
\begin{aligned}
I_{t}^{t}= & \beta_{0}+\beta_{1}\left(\frac{2 \chi}{r}+\frac{\chi^{\prime}}{\sqrt{h}} e^{-\lambda}\right)+\beta_{2}\left(\frac{\chi^{2}}{r^{2}}+\frac{2 \chi \chi^{\prime}}{r \sqrt{h}} e^{-\lambda}\right)-\left[\beta_{0}+3\left(\beta_{1}+\beta_{2}\right)\right] \frac{\chi^{2} \chi^{\prime}}{r^{2} \sqrt{h}} e^{-\lambda}, \\
I_{r}^{r}= & \beta_{0}+\beta_{1}\left(\frac{2 \chi}{r}+\sqrt{h} e^{-v}\right)+\beta_{2}\left(\frac{\chi^{2}}{r^{2}}+\frac{2 \sqrt{h} \chi}{r} e^{-v}\right)-\left[\beta_{0}+3\left(\beta_{1}+\beta_{2}\right)\right] \frac{\sqrt{h} \chi^{2}}{r^{2}} e^{-v}, \\
I_{\theta}^{\theta}= & I_{\phi}^{\phi} \\
= & \beta_{0}+\beta_{1}\left(\frac{\chi}{r}+\frac{\chi^{\prime}}{\sqrt{h}} e^{-\lambda}+\sqrt{h} e^{-v}\right)+\beta_{2}\left(\frac{\chi \chi^{\prime}}{r \sqrt{h}} e^{-\lambda}+\frac{\chi \sqrt{h}}{r} e^{-v}+\chi^{\prime} e^{-\lambda-v}\right) \\
& -\left[\beta_{0}+3\left(\beta_{1}+\beta_{2}\right)\right] \frac{\chi \chi^{\prime}}{r} e^{-\lambda-v} .
\end{aligned}
$$

Then the new constraints have the following forms

$$
\begin{aligned}
0=\nabla_{\mu} I_{r}^{\mu}= & \frac{\chi^{\prime}}{r^{2}} e^{-\lambda-v}\left\{\left(\beta_{0}+3\left(\beta_{1}+\beta_{2}\right)\right) \chi\left(2-2 e^{\lambda}+\chi v^{\prime} e^{v}\right)\right. \\
& -\beta_{1}\left[\left(\frac{2}{r}+v^{\prime}\right) e^{-\lambda}-\frac{2}{r}\right] r^{2} e^{\lambda+v} \\
& \left.-2 \beta_{2}\left[r\left(1-e^{\lambda}\right)+\chi\left(1-e^{\lambda}+r v^{\prime}\right) e^{v}\right]\right\} .
\end{aligned}
$$

\section{Numerical Results for minimal model}

From the requirement that vacuum configuration gives flat solution, we should have

$$
I_{\mu \nu}\left(\sqrt{g^{-1} f}\right)=\left(\beta_{0}+3 \beta_{1}+3 \beta_{2}+\beta_{3}\right) \eta_{\mu v}=0 .
$$

This gives a relationship between free parameters as $\beta_{0}+3 \beta_{1}+3 \beta_{2}+\beta_{3}=0$. In this report we consider the minimal model for the simplicity $\beta_{0}=3, \beta_{1}=-1$ and the graviton mass equals to cosmological constant $m_{0}^{2}=\Lambda$. This tells higher polynomials vanish. Moreover we use a boundary conditions that massive gravity solution have the same radius and central density with those in general relativity. If we use the SLy model as the equation of state [3], we obtain numerical results given in Fig.[1]. The figure shows the maximum mass of minimal model in the dRGT massive gravity is smaller than that in the general relativity. Therefore the minimal model is not suitable for astrophysical phenomenology.

\section{Conclusion}

We have shown that the minimal model in the dRGT massive gravity with flat reference metric is not favored for the explanation of massive neutron star. Actually in our paper [四], the result presented here has been checked also for other equations of state applied to quark stars. This is consistent with the literature [5], which states that the minimal model does not have the Vainshtein mechanism. However, there are still possibilities that non-minimal dRGT massive gravity can posses solutions similar to those in the general relativity. To obtain solutions in the non-minimal dRGT massive gravity, we have to check the discriminant of the forth-order algebraic constraint equations with respect to $\chi$. Moreover, we should reveal the relation between free parameters $\beta_{n}$ and the maximal mass. 


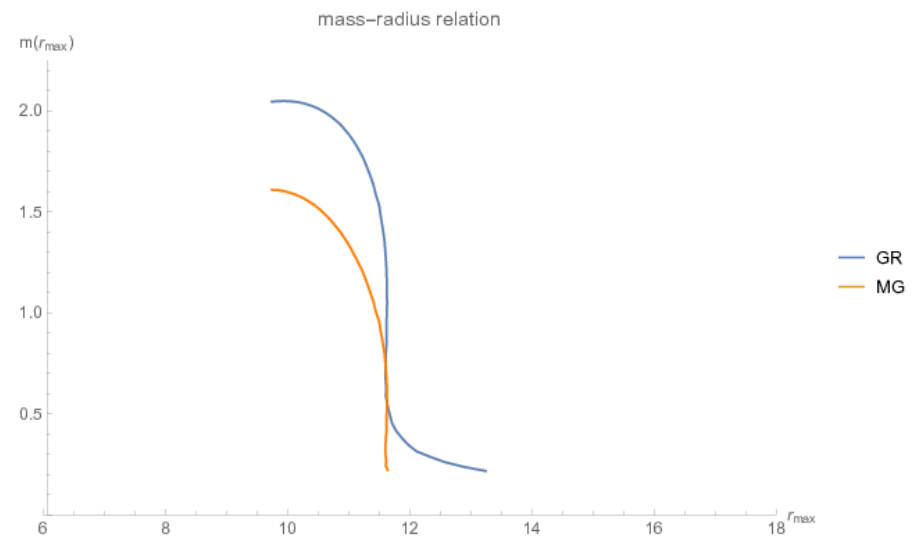

Figure 1: Mass-radius relations in SLy model

\section{Acknowledgments}

This research is supported in part by MEXT KAKENHI Grant-in-Aid for Scientific Research on Innovative Areas "Cosmic Acceleration" (No. 15H05890) and the JSPS Grant-in-Aid for Scientific Research (C) \# 23540296(S.N.), by the Grant-in-Aid for JSPS Fellows \# 15J06973(T.K.) and by MINECO (Spain) project FIS2013-44881 and I-LINK 1019 and JSPS fellowship ID No.: S15127 (S.D.O.).

\section{References}

[1] S. Capozziello, M. D. Laurentis, R. Farinelli, S. D. Odintsov, The Mass-Radius relation for Neutron Stars in $f(R)$ gravity, Phys. Rev. D 93 (2016) 023501

[2] C. de Rham, G. Gabadadze, A. J. Tolley, Resummation of Massive Gravity, Phys. Rev. Lett. 106 (2011) 231101 [hep-th/1011.1232]

[3] F. Douchin and P. Haensel A unified equation of state of dense matter and neutron star structure, Astronomy and Astrophysics 380 no. 1 (2001) 151 - 167

[4] T. Katsuragawa, S. Nojiri, S. D. Odintsov, M. Yamazaki, Relativistic stars in de Rham-Gabadadze-Tolley massive gravity, Phys. Rev. D 93 (2016) 124013 [gr-qc/1512 00660 ]

[5] S. Renaux-Petel On the Vainshtein mechanism in the minimal model of massive gravity, JCAP 1403 (2014) 043 [hep-th/1401.0497] 